\title{
Keiser, Juliane (2020). Zwischen Märchen, Tatsachenbericht und Glaubenszeugnis. Biblische Geschichten im Religionsunterricht der Grundschule. Göttingen: Vandenhoeck \& Ruprecht. ISBN 978-3-847 I-I I 20-7. 538 Seiten.
}

\section{Elisabeth Hennecke}

Katholische Don-Bosco-Schule Bochum (elisabeth.hennecke@uni-due.de)

Juliane Keiser stellt sich in ihrer Untersuchung einer enormen Herausforderung: Anhand videografierter Unterrichtsstunden aus drei Unterrichtsreihen erforscht sie das biblische „Konzept" der ExodusErzählung, das „explizit“ oder „implizit“ in den Unterricht eingebracht wird und den Schüler*innen „Deutungsspielräume“ eröffnen soll (27). Dieser Herausforderung stellt sie sich vor dem Hintergrund einer weiterhin sehr schmalen empirischen Datenlage über bibeldidaktische Umsetzungen im Religionsunterricht (der Grundschule). Sie leitet ihr Projekt aus fundierten, z. T. aus dem anglo-amerikanischen Bereich entnommenen, sowohl exegetischen als auch entwicklungspsychologischen Grundlegungen ab. Dabei überzeugen ihre Überlegungen zum Wesen biblischer Erzählungen, insbesondere zum Verhältnis von Realität und Fiktionalität, zur Bedeutung und Funktion von Erzählungen sowie zur Frage nach der Wahrheit biblischer Texte. Diese münden in eine Umschreibung des Wesens biblischer Texte, bei denen die „Wahrnehmung als Narrationen, als (fiktionale) Erzählungen keinen Mangel oder eine Schmälerung ihrer Glaubwürdigkeit darstellt“, sondern eine „einzigartige Möglichkeit bietet, Glaubenszeugnisse zu verdichten, zu teilen, an ihnen Teil haben zu lassen, sie zu bewahren und sie weiterzugeben“ (99). Diese Zeugnisse stehen nicht als „Dokumentation historischer Fakten“, sondern für einen „persönlichen und existentiellen Wahrheitsanspruch“ (98).

Auch in der Darstellung der Rezeptionsvoraussetzungen der Schüler*innen werden wichtige Grundlagen der sich verändernden Forschungslage benannt, insbesondere die Bedeutung von bereichsspezifischen Voraussetzungen und domänenabhängiger Wissensbestände, bei denen davon ausgegangen wird, dass die Weiterentwicklung der individuellen Vorstellungshorizonte durch externe Einflüsse angeregt werden kann. Auch der Bezug auf die Entwicklung des Geschichtsbewusstseins bei Heranwachsenden liefert wichtige Erkenntnisse für die gewählte Fragestellung. Hilfreiche Impulse hätte eventuell noch ein Rekurs auf den Stand der Bibeldidaktik liefern können. Dieser Bezug wird am Ende der Arbeit hergestellt, wo die Untersuchungsergebnisse im Licht bibeldidaktischer Erkenntnisse ausgewertet werden. Die Zielsetzung der Untersuchung erfährt dadurch an später Stelle eine Erweiterung: in der von Berg geäußerten Kritik an einer „unreflektiert normativen Präsentationsweise“ (508). Die Zielsetzung der Untersuchung wird ansonsten im einleitenden Kapitel dargestellt, wo sich die theoretischen Bezüge noch nicht vollständig erschließen. Jedoch wird das erkenntnisleitende Interesse in der Beschreibung und in der Auswertung der Untersuchung so deutlich und durchgängig aufgegriffen, dass es als Zielsetzung stets erkennbar ist.

Im Untersuchungsteil werden sowohl die methodologische Grundentscheidung als auch das methodische Inventar sorgfältig abgewogen, begründet eingesetzt und professionell umgesetzt. So werden 38 Religionsunterrichtsstunden in drei dritten Klassen bei verschiedenen Lehrkräften videografiert und durch teilnehmende Beobachtung aufgezeichnet, anschließend gründlich transkribiert und in Auswertungseinheiten definiert (Einführungsstunden und incidents). Diese werden anhand der dokumentarischen Methode sequenzanalytisch ausgewertet, zusammengefasst und verglichen. Da die Auswertung sowohl die Darstellungsweise der Lehrkräfte als auch thematische Schwerpunkte der biblischen Auseinandersetzung im Unterricht sowie die möglichen Konzeptentwicklungen auf Seiten der Kinder um- 
fasst, ist die Auswertungsbeschreibung sehr umfangreich und detailliert, stellt kontinuierlich Rückbezüge zum Erhebungsmaterial her und wird immer wieder auf den Forschungsschwerpunkt fokussiert. Dadurch gelingt eine große Verständlichkeit und gute Nachvollziehbarkeit der Forschungsergebnisse. Durch dieses gestufte Auswertungsverfahren und die steten Rückgriffe auf das konkrete Material entstehen zeitweise Längen und Dopplungen, die an manchen Stellen durch eine Reduktion zu einer distanzierteren Analyse beigetragen hätten.

Insgesamt eröffnet die vorliegende Untersuchung jedoch einen äußerst interessanten Blick und wichtige Einsichten zum Umgang mit biblischen Texten im Religionsunterricht: Durchgängig ist die Tendenz der Lehrkräfte zu erkennen, die biblischen Texte auf ihre realen Hintergründe zurückzuführen und sie damit in die Nähe von Tatsachenberichten zu bringen. Die Lehrerinnen thematisieren nicht selten Argumentationszusammenhänge, bei denen sie sich auf reale Gegebenheiten berufen, z. B. Israeliten als ägyptische Sklaven, Manna als „Tamariskenmanna“, ... Gleichzeitig werden die alttestamentlichen Erzählungen immer wieder wie fiktionale Texte eingebracht, behandelt und angesprochen, wenn „Geschichten erzählt" werden, die ein „Happy End“ haben, in einer ",anderen Zeit“ spielen und märchenhafte Züge tragen. Im untersuchten Unterricht herrschen die von diesen beiden Ambivalenzen geprägten Konzepte vor, die sich auch in den sehr selten festzustellenden Rezeptionen der Kinder widerspiegeln. Eine weitere Funktion der Bibel ist die der Argumentationsverstärkung und Autoritätsvermittlung (,,so steht es in der Bibel“" 468). An keiner Stelle des Unterrichts werden die biblischen Texte als Texte eines Glaubenszeugnisses dargestellt, die ihr „Wesen“ auszeichnen, weil sie in einer bestimmten geschichtlichen und sozialen Situation entstanden, von einer religiösen Überzeugung der Verfasser*innen geprägt sind und den Adressat*innen zu einer bestimmten Rezeption herausfordern. Von Seiten der Schüler*innen gibt es wenig konfliktträchtige Anfragen; auch scheinen wundersame Elemente wenig zu Fragen herauszufordern. Häufig verbleiben die Auseinandersetzungen der Kinder auf einer realitätsbezogenen Ebene, was von den Lehrkräften in der Regel unterstützt wird, z. B. in der Frage, ob das Rote Meer rot oder blau zu zeichnen sei.

In den Zusammenfassungen, der abschließenden Auswertung und im Ausblick verbindet die Forscherin die vorangestellten theoriegeleiteten Erkenntnisse mit ihren Untersuchungsergebnissen sowie bibeldidaktischen Desideraten und kommt zu nachvollziehbaren, aufschlussreichen Erkenntnissen sowie zu sinnvoll abgeleiteten Konsequenzen. Sie benennt sich eröffnende Anschlussfragen und vor allem die Notwendigkeit, das Wesen biblischer Texte den Lehrkräften in Fort- und Ausbildung deutlicher zu vermitteln, um textspezifischere Auseinandersetzungen im Unterricht zu ermöglichen. Diese könnten dazu beitragen, entwicklungsbedingte Widersprüche zu verhindern, die bei einer entweder an der Realität oder der Fiktionalität orientierten Vermittlung von biblischen Texten schnell entstehen. Das Anliegen, biblische Texten stärker als Glaubenstexte in den Unterricht einzubringen, erhält eine zunehmende Bedeutung, da der Religionsunterricht immer häufiger den Erstkontakt mit biblischen Texten und somit eine bedeutsame Einflussgröße für die Entwicklung des domänenspezifischen biblischen Wissens darstellt.

Über diese Erkenntnisgewinne hinaus zeichnet sich diese Untersuchung durch einen hohen Innovationsgrad aus, weil es kaum Untersuchungen in diesem Bereich gibt, und schafft einen unverstellten Blick auf den Unterrichtsalltag in der Grundschule, was auch der Tatsache geschuldet ist, dass die Verfasserin selbst in der Schule tätig ist. Sie schafft eine durchgängig bewertungsfreie Darstellung des Unterrichtsgeschehens, zeichnet ein aufschlussreiches, realistisches Bild vom Religionsunterricht in der Grundschule und legt in ihrer Forschungsarbeit fundiert und begründet Optimierungsmöglichkeiten desselben dar. 